\title{
Conjugacy of Coxeter elements
}

\author{
HENRIK ERIKSSON* and Kimmo ERIKSSON ${ }^{\dagger}$ \\ Submitted: Sep 29, 2008; Accepted: Feb 26, 2009; Published: Mar 6, 2009
}

Mathematics Subject Classification: 20F55

\begin{abstract}
For a Coxeter group $(W, S)$, a permutation of the set $S$ is called a Coxeter word and the group element represented by the product is called a Coxeter element. Moving the first letter to the end of the word is called a rotation and two Coxeter elements are rotation equivalent if their words can be transformed into each other through a sequence of rotations and legal commutations.

We prove that Coxeter elements are conjugate if and only if they are rotation equivalent. This was known for some special cases but not for Coxeter groups in general.
\end{abstract}

\section{Introduction}

Consider the Coxeter group defined by the Coxeter graph below. A Coxeter word is a list of all generators in any order, so there are 24 Coxeter words in our example. Interpreting words as products we get 12 different Coxeter elements $\left(s_{0}\right.$ commutes with $s_{2}$ and $\left.s_{3}\right)$, which fall into two different conjugacy classes.

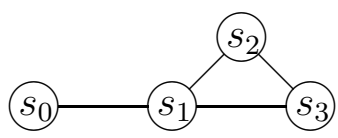

Conjugation by the first letter of a Coxeter word will have the effect of moving this letter to the end of the word. For example, if $w=s_{0} s_{1} s_{2} s_{3}$ then $s_{0} w s_{0}=s_{1} s_{2} s_{3} s_{0}$. We call this a rotation of the word. Say that two words are rotation equivalent if one can be obtained from the other by a series of rotations and commutations. For example,

$$
s_{0} s_{1} s_{2} s_{3} \sim s_{1} s_{2} s_{3} s_{0} \sim s_{1} s_{2} s_{0} s_{3} \sim s_{2} s_{0} s_{3} s_{1}
$$

Our aim is to prove the following characterization of conjugacy of Coxeter elements.

\footnotetext{
*School of Computer Science and Communication, Royal Institute of Technology, SE-10044 Stockholm, Sweden. email: henrik@nada.kth.se

${ }^{\dagger}$ School of Education, Culture and Communication, Mälardalen University, Box 883, SE-72123 Västerås, Sweden. email: kimmo.eriksson@mdh.se
} 
Theorem 1.1. Coxeter elements are conjugate if and only if they are rotation equivalent.

We stated this result at the FPSAC meeting in 1994, but gave proofs only for the two important special cases when the Coxeter graph is a tree or a cycle (covering all finite and affine groups). For these special cases, the result has since been rediscovered by Shi [4], who extended it to cycles with trees attached. Here we present the first proof of the general result.

\section{Edge orientations and chip-firing}

For a graph $G$, an acyclic edge orientation is an assignment of directions to all edges, such that the resulting digraph is acyclic. This is always possible. A simple observation is that the resulting digraph contains at least one sink, i.e. a vertex with no outgoing edges.

If each arrowhead is detached and pronounced a chip, we get a distribution of chips on the vertices and can play the chip-firing game introduced in [2]. Translated into edge orientations, a legal move consists in choosing a sink and firing it, that is changing it into a source by reversing all its edges. Since neither sinks nor sources belong to any cycles, the graph will still be acyclic and contain a sink, so the game goes on forever.

Several authors have rediscovered and analysed this edge reorientation game. All the following facts can be derived from the work of Pretzel [5], but for convenience we give a self-contained presentation.

Proposition 2.1. If a vertex $s$ is fired in an acyclic edge orientation, there is a continuation in which every other vertex is fired exactly once. Such a game sequence restores the original edge orientation.

Proof. Induction over the number of vertices proves the proposition: After firing $s$, use the induction hypothesis to fire all remaining nodes. The base case is trivial as is the restoration of original orientation.

Corollary 2.2. There is a play sequence from $u$ to $v$ if and only if there is a play sequence from $v$ to $u$.

Proof. If a single move can be inverted, so can a sequence of moves. Thus, it is sufficient to consider the case when $v$ is the result of firing a single vertex in position $u$, so the proposition applies.

According to this result, reachability of positions in this game constitutes an equivalence relation that partitions acyclic edge orientations into reachability classes. For many graphs, it is now a rather simple matter to enumerate acyclic edge orientations and reachability classes. Two basic cases are covered by our next proposition.

Proposition 2.3. For a tree with $n$ nodes, there are $2^{n-1}$ acyclic edge orientations but only one reachability class. For an $n$-cycle, there are $2^{n}-2$ acyclic edge orientations and $n-1$ reachability classes of sizes $\left(\begin{array}{l}n \\ 1\end{array}\right), \ldots,\left(\begin{array}{c}n \\ n-1\end{array}\right)$. 
Proof. An $n$-vertex tree has got $n-1$ edges with no restrictions on orientations, for all directed trees are acyclic. The statement that all edge orientations are reachable from each other has a simple induction proof: choose a leaf, play to give the rest of the tree the desired orientation, firing the leaf when necessary, finally fire the chosen leaf once more if needed to reorient its edge.

For an $n$-cycle, exactly two orientations are forbidden, namely all $n$ clockwise or all $n$ anti-clockwise. Consider the $\left(\begin{array}{l}n \\ k\end{array}\right)$ orientations with $k$ anti-clockwise edges. Firing a node may be seen as moving the anti-clockwise arrow one step forward, e.g. $\bullet \leftarrow \hookrightarrow-\hookrightarrow \bullet$ to

It is obvious that any position with $k$ anti-clockwise arrows can be reached in this way.

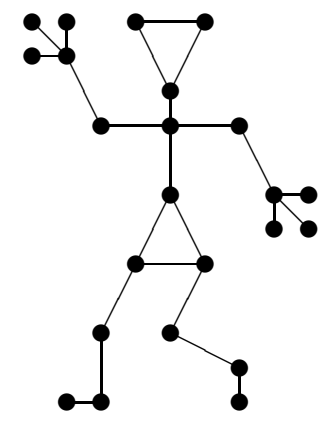

Figure 1: A trunk with four limbs and three joints.

A connected graph that is not a tree may be decomposed uniquely as a leafless trunk (the subgraph obtained by successive removal of leaves until none are left) and a collection of limbs, defined as trees that connect to the trunk at one vertex only, called a joint. We may then apply the same induction argument as we used for trees to obtain the following useful result.

Proposition 2.4. In an acyclic edge orientation, if edges on limbs are arbitrarily redirected, the result is a new acyclic orientation in the same reachability class.

\section{$3 \quad$ Words with intervening neighbours}

Let $G$ be the Coxeter graph of a Coxeter group with generators $S$. Consider a word $w$ in the alphabet $S$. If there is an edge between $s$ and $t$ and if the first occurrence of $s$ in $w$ precedes the first occurrence of $t$, we orient the edge like $s \rightarrow t$. In this way we edge-orient the subgraph of $G$ spanned by the letters in $w$.

Now, consider this edge orientation as a right-to-left process on the word $w$. The rightmost letter orients no edge, the two rightmost letters orient the edge between the corresponding vertices (if there is one) and the larger the segment, the more edges get oriented. When a letter $t$ reappears, we may have to reverse some arrows $s \rightarrow t$, namely 
when the new situation is $t \cdots s \cdots t \cdots$, and if all $t$-neighbours occur in between the first and the second $t$, this will be a chip-firing move.
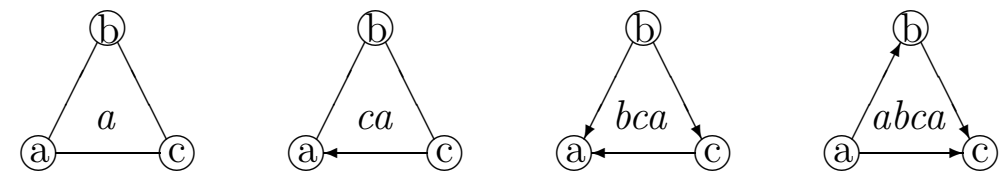

Figure 2: Successive edge orientation by the word abca

Definition 3.1. A word has the intervening neighbours property if any two occurrences of the same letter are separated by all its graph neighbours.

If $w$ has this property and if all letters of $S$ occur in $w$, eventually the right-to-left process will have oriented all edges in $G$, so giving such a word $w$ is equivalent to giving an initial edge orientation and a play sequence.

If two words, $w$ and $w^{\prime}$, represent the the same group element, are the corresponding edge orientations necessarily in the same reachability class? For a leafless graph the answer is yes.

Proposition 3.2. Let $G$ be a trunk without limbs and let $w$ be a word with the intervening neighbours property in which all letters of $S$ occur. Then $w$ is a reduced word for the group element it represents, all reduced words for this element are obtained by commutations in $w$ and all edge orientations defined by these words belong to the same reachability class.

Proof. When two occurrences of the same letter are separated by two or more neighbours, no braid relations such as sts $=t s t$ apply, so commutations are the only applicable rewriting rules. Commutations preserve the intervening neighbours property and no reduction is possible. Nor do commutations affect the edge orientation.

Now let $G$ be a general graph, regarded as a trunk with limbs. It is no longer true that the intervening neighbours property is an invariant under rewritings - for example, if $s$ is a leaf connected to the trunk vertex $t$, the braid transformation $s t s=t s t$ will produce two occurrences of $t$ with only one intervening neighbour. It turns out that only limb letters are involved in braid transformations and that the intervening neighbours property stays true for the other trunk letters, with a slight modification for the joints (vertices in which a limb connects with the trunk). Obviously, trunk letters never occur in higher braid transformations like stst $=t s t s$.

The following lemma states properties that are true for a word with the intervening neighbours property and which stay true under rewritings.

Lemma 3.3. The following word properties are invariant under commutations st $=$ ts and braid transformations sts $=$ tst.

- The intervening neighbours property holds for trunk letters that are not joints, i.e. any two occurrences of such a letter are separated by all its neighbours. 
- Two occurrences of the same joint are either separated by all its trunk neighbours (it has at least two) or by no trunk neighbour (but by at least one limb neighbour).

Proof. Invariance under commutations is trivial. A braid transformation must involve two limb letters (one of which may be a joint) so the first property stays true. If $s$ is a joint or $t$ is a joint, the second property still stays true after $s t s=t s t$.

We are now almost ready to extend Prop. 3.2 to trunks with limbs. The missing piece was provided by David Speyer [6].

Lemma 3.4 (Speyer, 2008). For infinite irreducible Coxeter groups, all words with the intervening neighbours property are reduced.

Speyer actually states the result for c-admissible sequences, that is valid play sequences from edge orientation $c$, but as we have noted, the concepts are equivalent.

Proposition 3.5. Let $G$ be any connected non-tree graph and let $w$ be a word with the intervening neighbours property in which all letters of $S$ occur. Then $w$ is a reduced word, all reduced words for this element are obtained by commutations in $w$ and braid transformations involving only limb letters, and all edge orientations defined by these words belong to the same reachability class.

Proof. All non-tree Coxeter graphs define infinite groups, so Speyer's result applies. Observe that, by Prop. 2.4, the orientation of limb edges is insignificant for reachability, and neither commutations nor braid transformations of limb letters influence the edge orientations in the trunk. Hence, we may disregard all limb letters except for the joints. According to Lemma 3.3, the intervening neighbours property with respect to the set of trunk letters that are not joints will hold under rewriting. Joints may duplicate, but as there are only limb neighbours between the duplicates, the argument in the proof of Prop. 3.2 goes through with respect to the trunk letters. When two occurrences of the same letter are separated by two or more neighbours, no braid relations of type sts $=t s t$ apply, so commutations are the only applicable rewriting rules. Commutations preserve the intervening neighbours property and no reduction is possible. Nor do commutations affect the edge orientation.

\section{Coxeter elements}

A Coxeter word has one instance of each letter, so it defines an acyclic orientation of the Coxeter graph. This orientation is in fact well defined by the Coxeter element, as all words representing the same element are obtainable by commutations. And in Prop. 2.1 we noted that for every acyclic orientation there is a play sequence in which all vertices are fired once, i.e. a corresponding Coxeter word.

So, Coxeter elements correspond bijectively to acyclic edge orientations of the Coxeter graph. And we have seen that moving the first letter of a Coxeter word to the end is the same as firing the corresponding vertex. This proves the following proposition from [3]. 
Proposition 4.1. Rotation of Coxeter words induces an equivalence relation on the set of Coxeter elements, that corresponds precisely to the reachability relation on the set of acyclic edge orientations.

Proof of theorem 1.1. Our main theorem states that reachability classes and conjugacy classes coincide. Rotating a letter $s$ from the beginning to the end is the same thing as conjugating by $s$, therefore rotation equivalent elements are indeed conjugate. Proving that two conjugate Coxeter elements $w$ and $w^{\prime}=u w u^{-1}$ must belong to the same reachability class is harder, but because of Prop. 2.3 we need only prove it for connected non-tree graphs.

The trick is to consider a power $\left(w^{\prime}\right)^{k}=u w^{k} u^{-1}$ with $k$ sufficiently large. Note that $\left(w^{\prime}\right)^{k}$ and $u w^{k} u^{-1}$ are two different words for the same group element, so the second one must be reducible. The proof will have three steps:

1. The word $\left(w^{\prime}\right)^{k}$ is reduced.

2. The word $u w^{k} u^{-1}$ has a reduced form $u_{1} w u_{2}$.

3. Since $w$ and $w^{\prime}$ appear in words representing the same element, they belong to the same reachability class and are rotation equivalent (as explained in detail below).

We regard the graph as a trunk with limbs. The proof is easiest if there are no limbs, but in the end, the limbs will turn out to be of no consequence.

1. Since $\left(w^{\prime}\right)^{k}$ has the intervening neighbours property, Prop. 3.5 implies that $\left(w^{\prime}\right)^{k}$ is reduced,

2. The well-known deletion property for Coxeter groups (see [1]) states that any word can be brought to a reduced form through a series of successive deletions of pairs of letters (not necessarily adjacent). For $u w^{k} u^{-1}$, the number of such deletions is the same as the number of letters in $u$. For any $k$, greater than this number, at least one instance of $w$ will remain intact after the deletions.

3. Prop. 3.5 tells us that all edge orientations obtained from the words $\left(w^{\prime}\right)^{k}$ and $u_{1} w u_{2}$ by the right-to-left process are in the same reachability class. When the sequence $w$ in the middle of the second word has just been processed, the edge orientation is of course completely defined by $w$. Therefore $w^{\prime}$ and $w$ are rotation equivalent.

Note. Pretzel [5] showed that two acyclic orientations belong to the same class if they have the same circulation around every cycle, so we have a very explicit characterization of the conjugacy classes.

Acknowledgments. We thank Henning Mortveit and Matthew Macauley for urging us to publish the lost proof. 


\section{References}

[1] A. Björner and F. Brenti, Combinatorics of Coxeter Groups, Grad. Texts in Math., vol. 231, Springer, 2005.

[2] A. Björner, L. Lovász and P. W. Shor, Chip-firing games on graphs, European J. Combin. 12, 1991, 283-291.

[3] H. Eriksson and K. Eriksson. Chip-firing and Coxeter elements. In Proceedings of the 6th conference on Formal Power Series and Algebraic Combinatorics, 145-151, DIMACS, 1994.

[4] Jian Yi Shi, Conjugacy relation on Coxeter elements, Advances in Mathematics 161, 2001, 1-19.

[5] O. Pretzel, On reorienting graphs by pushing down maximal vertices, Order 3, 1986, no. 2, 135-153.

[6] D. Speyer, Powers of Coxeter elements in infinite groups are reduced, Proc. Amer. Math. Soc. 137, 2009, 1295-1302 